\title{
Preparation and Characterization of R.F. Magnetron Sputtered Mo:ZnO Thin Films
}

\author{
K. Srinivasarao, ${ }^{1}$ G. Srinivasarao, ${ }^{1}$ K. V. Madhuri, ${ }^{1}$ \\ K. Krishna Murthy, ${ }^{2}$ and P. K. Mukhopadhyay ${ }^{3}$ \\ ${ }^{1}$ Department of Science and Humanities, Vignan University, Vadlamudi, Andhra Pradesh 522 213, India \\ ${ }^{2}$ Postgraduate Department of Physics and Electronics, P.B. Siddhartha College of Arts and Science, Vijayawada, \\ Andhra Pradesh 520 010, India \\ ${ }^{3}$ Laboratory for Condensed Matter Physics, Satyendra Nath Bose National Centre for Basic Sciences, Salt Lake, Kolkata 700 098, India
}

Correspondence should be addressed to K. Srinivasarao; kotarisrinu@yahoo.co.in

Received 17 June 2013; Accepted 31 July 2013

Academic Editors: K. Kalantar-Zadeh and H. Leiste

Copyright (c) 2013 K. Srinivasarao et al. This is an open access article distributed under the Creative Commons Attribution License, which permits unrestricted use, distribution, and reproduction in any medium, provided the original work is properly cited.

The $\mathrm{ZnO}$ and $\mathrm{Mo}: \mathrm{ZnO}$ thin films were deposited by radio frequency magnetron sputtering on quartz and intrinsic silicon (100) substrates at a fixed combined partial pressure $1 \times 10^{-2}$ mbar of $\mathrm{Ar}+\mathrm{O}_{2}$ and substrate temperatures of $473 \mathrm{~K}$ and $673 \mathrm{~K}$. The effect of Molybdenum doping on $\mathrm{ZnO}$ thin films with different Molybdenum concentrations (1-2 atomic percent) was studied with the help of structural and microstructural characterization techniques. The films deposited at a substrate temperature of $473 \mathrm{~K}$ exhibited strong $c$-axis orientation with predominant (002) peak. At $673 \mathrm{~K}$, along with (002) orientation, other orientations (100), (101), (220), and (103) were also observed. Among these, the (220) peak indicates the cubic phase of $\mathrm{ZnO}$. With increasing Molybdenum concentration, the cubic phase of $\mathrm{ZnO}$ disappeared, and the (002) orientation became strong and intense. The composition analysis reveals that the undoped $\mathrm{ZnO}$ films deposited at $473 \mathrm{~K}$ have oxygen deficiency, and the ratio of $\mathrm{Zn} / \mathrm{O}$ is improved with increasing Mo atomic percent in $\mathrm{ZnO}$. The surface morphological features reveal that the undoped $\mathrm{ZnO}$ films were found to be uniform and have grain size of around $30 \mathrm{~nm}$. The optical energy gap of the undoped $\mathrm{ZnO}$ films is $3.05 \mathrm{eV}$ and increases with increasing Mo concentration. The thickness of the films is around $456 \mathrm{~nm}$.

\section{Introduction}

For the past few years, there has been a lot of interest shown to find new oxide materials which will act as transparent as well as conducting materials. Many metal oxides and their compounds have been found to be transparent and conductve. Among these oxides indium tin oxide (ITO) and aluminum doped zinc oxide (AZO) were proved to be transparent and conducting materials [1-3]. Still there has been a lot of interest shown to overcome the problem involved with those materials like abundance of material, stability, performance, reliability, and durability. So the search for new transparent and conducting oxides continues in the years to come. Various metallic elements doped to $\mathrm{ZnO}$ were tested for their transparency in the wavelength range from $450 \mathrm{~nm}$ to $750 \mathrm{~nm}$ for conductivity. The principal elements doped to $\mathrm{ZnO}$ were $\mathrm{Al}, \mathrm{In}, \mathrm{Sn}, \mathrm{Ga}, \mathrm{Cd}$ [4], and Mo. Among these, an extensive work was done on $\mathrm{Al}$ doped $\mathrm{ZnO}$ films, and they were proved as potential transparent conductors. Among these, Molybdenum doped $\mathrm{ZnO}$ was also found to be an attractive candidate as transparent conducting oxide (TCO) due to its ease to doping to $\mathrm{ZnO}$.

Moreover, the difference between the valence electrons of $\mathrm{Mo}^{6+}$ and $\mathrm{Zn}^{2+}$ is 4 . This difference can produce enough free carriers and reduce the ion scattering effect for a very small amount of Mo [5]. Moreover, Mo exhibits multiple valence states of $+6,5,4,3,2$. This enables the contribution of multiple carriers by a single Mo dopant atom [6].

The work reported on $\mathrm{ZnO}$ is extensive, and also the significant work was reported on $\mathrm{Mo}: \mathrm{ZnO}[7,8]$. In TCOs, the concentration of dopant element to host compound decides the transparency and conductivity. In general, the conductivity increases with increasing dopant concentration, but at the same time it reduces the transparency. So, 
the optimization of deposition parameters like argon and oxygen pressure (in case of sputtering) during deposition and substrate temperature will decide the proper doping of dopant in the host lattice which in turn will result in the thin films with high transparency and conductivity. In the present investigation, the effect of Mo doping on various physical properties like structure, surface morphology, and optical properties of $\mathrm{ZnO}$ was studied in detail. Deposition of oxide films by r.f. sputtering has its own advantage over other physical vapour deposition techniques. The chief advantage is the control on deposition parameters like oxygen, Ar pressure, and substrate temperatures. The $\mathrm{ZnO}$ and $\mathrm{Mo}: \mathrm{ZnO}$ films were deposited at various sputtering pressures $\left(1 \times 10^{-2}\right.$ and $1 \times 10^{-5} \mathrm{mbar}$ ) and substrate temperatures of $473 \mathrm{~K}$ and $673 \mathrm{~K}$. In the present work, the physical properties of $\mathrm{ZnO}$ and $\mathrm{Mo}: \mathrm{ZnO}$ thin films for various concentrations of Mo thin films deposited at the sputtering pressure of $1 \times 10^{-2} \mathrm{mbar}$ and at a substrate temperatures of $473 \mathrm{~K}$ and $673 \mathrm{~K}$ were reported.

\section{Materials and Methods}

Thin films of $\mathrm{ZnO}$ and $\mathrm{Mo:} \mathrm{ZnO}$ were deposited by r.f. magnetron sputtering using Hind Hivacuumcoating unit. High pure ZnO powder (Sigma Aldrich, 99.99\%) was used to prepare pellet of $2.54 \mathrm{~cm}$ (one inch) in diameter, and the prepared pellet was sintered at a temperature of $1073 \mathrm{~K}$ and used as target for deposition of the films. In the same way $\mathrm{MoO}_{3}$ was added to $\mathrm{ZnO}$ to maintain Mo composition at 1 at.\% in $\mathrm{ZnO}$. Three separate pellets were prepared by maintaining at.\% of Mo at 1.5 and 2 and sintered at $973 \mathrm{~K}$. The reason for sintering the $\mathrm{MoO}_{3}$ contained $\mathrm{ZnO}$ pellets at different temperature is $\mathrm{MoO}_{3}$ sublime at around $973 \mathrm{~K}$. The vacuum coating unit was initially evacuated to a base pressure of $1 \times 10^{-6}$ mbar; then Argon gas was allowed to flow into the chamber. The discharge was produced by applying a voltage of around $400 \mathrm{~V}$. Thereafter $\mathrm{O}_{2}$ was introduced in the chamber using mass flow meter, and the voltage was so adjusted to attain a sputtering power of $50 \mathrm{~W}$. For all film depositions, Ar: $\mathrm{O}_{2}$ flow ratio was maintained at $5: 2$. The films were deposited at a fixed sputtering pressure of $1 \times 10^{-2} \mathrm{mbar}$ and at different substrate temperatures of $473 \mathrm{~K}$ and $673 \mathrm{~K}$. The target to substrate distance is $5 \mathrm{~cm}$. The thickness of the target is $3 \mathrm{~mm}$. The substrates used are silicon and glass and are square $(5 \mathrm{~mm} \times 5 \mathrm{~mm})$ in shape. The substrates were kept at zero potential. The grazing incidence XRD (GIXRD) of the films at a glancing angle of 0.5 degree was performed by panalytical X-ray diffractometer. The surface microstructure was performed by using FIB quanta dual beam SEM (Helios Nova Nano lab 600). The optical transmittance spectra of the films were performed by UV-VIS spectrophotometer (UV-2450 SHIMADZU make) in the wavelength range 300$700 \mathrm{~nm}$. The spectral resolution of the spectrophotometer is $0.2 \mathrm{~nm}$. The thicknesses of the films were measured using spectroscopic ellipsometer (model EC-400 and M2000D, J.A. Woollam Co., inc make). The spectral resolution of the ellipsometer is $0.5 \mathrm{~nm}$. The growth rate is $8 \mathrm{~nm}$ per minute. All the films have thickness around $456 \mathrm{~nm}$.

\section{Results and Discussion}

3.1. Compositional Analysis. The chemical composition of zinc and oxygen in $\mathrm{ZnO}$ and Molybdenum in Mo: $\mathrm{ZnO}$ effects the physical properties of $\mathrm{ZnO}$ and $\mathrm{Mo}: \mathrm{ZnO}$ thin films. In the present investigation the chemical composition of $\mathrm{Mo}, \mathrm{Zn}$, and oxygen in Mo: $\mathrm{ZnO}$ films was found by EDAX spectroscopy. The chemical composition of the $\mathrm{ZnO}$ and $\mathrm{Mo}: \mathrm{ZnO}$ films deposited at the sputtering pressure of $1 \times 10^{-2}$ mbar and at a substrate temperature of $473 \mathrm{~K}$ was obtained by EDAX. The undoped $\mathrm{ZnO}$ films have zinc of 54.3 at.\% and Oxygen 45.7 of at.\%, which indicates that the films have oxygen deficiency. The atomic percentage $\left(C_{\mathrm{A}}\right)$ of the element in the matrix is calculated by the formula [9]:

$$
C_{\mathrm{A}}=\frac{S_{\mathrm{A}} I_{\mathrm{A}}}{\sum S_{i} I_{i}},
$$

where $S_{\mathrm{A}}$ and $I_{\mathrm{A}}$ are the EDAX sensitivity factors and integral count of element $\mathrm{A}$, respectively. In the present case $i$ is equal to 3 since $\mathrm{Mo}: \mathrm{ZnO}$ films contain three elements. In the present case the at.\% of the different elements in the film is obtained from the instrument calculated by the above formula.

The Molybdenum content in the $\mathrm{Mo}(1.5 \%): \mathrm{ZnO}$ films deposited at the above conditions is 1.48 at.\% which is almost equal to the molybdenum contained in the target. With the increasing of molybdenum at.\% to 2 in the target, the molybdenum at.\% present in the film becomes 2.15 which is slightly above the molybdenum content in the target. At the same time the atomic ratio of $\mathrm{Zn} / \mathrm{O}$ was improved in case of molybdenum doped films. As a result, the molybdenum doped $\mathrm{ZnO}$ films have good proportion of $\mathrm{Zn} / \mathrm{O}$ ratio when compared to the undoped $\mathrm{ZnO}$. This indicates that when a high oxidation state metal is doped to the $\mathrm{ZnO}$, the oxygen deficiency can be improved. The composition of zinc, oxygen, and molybdenum in $\mathrm{Mo}: \mathrm{ZnO}$ thin films deposited at $1 \times$ $10^{-2}$ mbar and at $473 \mathrm{~K}$ is given in Table 1.

3.2. Structural Analysis. The crystal structure of $\mathrm{ZnO}$ and $\mathrm{Mo}: \mathrm{ZnO}$ films was determined by XRD spectra obtained by grazing incidence $\mathrm{X}$-ray diffraction. The X-ray diffraction spectra of the $\mathrm{ZnO}$ and $\mathrm{Mo}: \mathrm{ZnO}$ films deposited at $1 \times$ $10^{-2}$ mbar and at a substrate temperature of $473 \mathrm{~K}$ are shown in Figure 1.

The $\mathrm{Mo}(0 \%): \mathrm{ZnO}$ films were observed to be $c$-axis oriented films with strong (002) orientation, which indicates the hexagonal wurtzite structure of $\mathrm{ZnO}$ [10]. It was observed that another crystallographic orientation (220) indicates cubicphase $\mathrm{ZnO}$ [11]. The $2 \theta$ of the (002) peak shifts towards higher value with increasing molybdenum concentration and indicates reduction of inter-planar spacing " $d$ " in the films. In case of 1 at.\% molybdenum doping, the intensity of (002) peak is low when compared with that of the (103) peak. It is also observed that the (002) peak is unsymmetric with molybdenum doping. The $c$-axis preferential orientation of the films is unchanged even with increasing molybdenum concentration. Also the crystallographic (002) peak becomes broad with increasing molybdenum concentration. The literature reveals 
TABLE 1: EDAX data of Mo:ZnO films.

Atomic percent of $\mathrm{Mo}$ in $\mathrm{ZnO}$

\begin{tabular}{lccc} 
& At.\% of oxygen & At.\% of zinc & At.\% of molybdenum \\
\hline $\mathrm{Mo}(0 \%): \mathrm{ZnO}$ & 45.71 & 54.29 & 0 \\
$\mathrm{Mo}(1.5 \%): \mathrm{ZnO}$ & 46.4 & 52.11 & 1.48 \\
$\mathrm{Mo}(2 \%): \mathrm{ZnO}$ & 46.81 & 51.04 & 2.15 \\
\hline
\end{tabular}

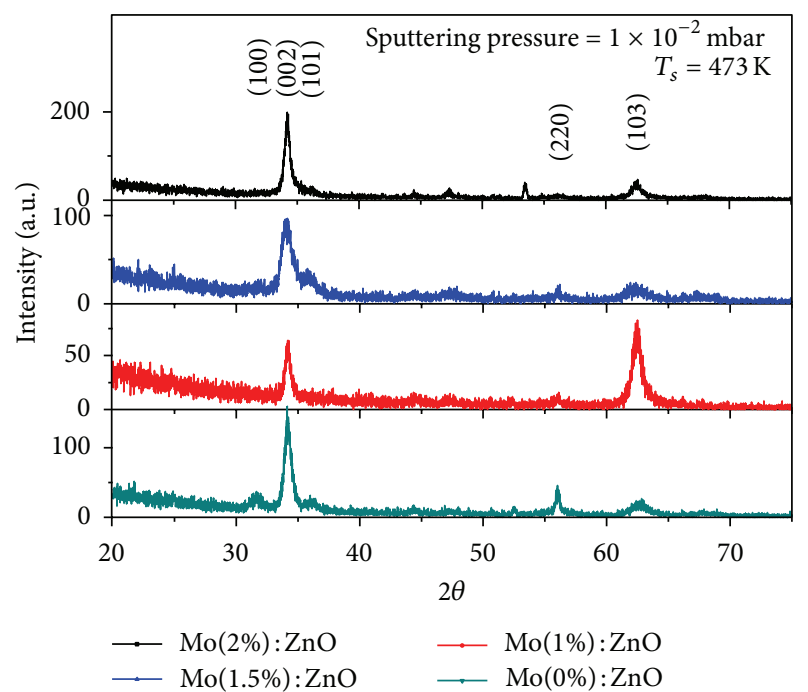

FIgURE 1: X-ray diffraction spectra of the Mo: $\mathrm{ZnO}$ thin films deposited at a fixed sputtering pressure of $1 \times 10^{-2} \mathrm{mbar}$ and at a substrate temperature of $473 \mathrm{~K}$.

that the reduction in the intensity of the crystallographic peak is attributed to the reduction in the crystallinity of the films $[3,12]$. In the present case we have observed the peak intensity variation that may not be completely due to Mo doping but also due to variation in thickness of the films.

The XRD spectra of the films deposited at $1 \times 10^{-2} \mathrm{mbar}$ and at $673 \mathrm{~K}$ are shown in Figure 2. The $\mathrm{Mo}(0 \%): \mathrm{ZnO}$ films exhibit (002) peak, which indicates that the $\mathrm{ZnO}$ films were crystallized in hexagonal wurtzite phase. The other orientations, (100) and (101), were also observed along with (002), and intensity of these orientations is comparable with that of (002). The (220) orientation at $2 \theta=56.2$ corresponds to cubic $\mathrm{ZnO}$ which indicates that the films contain wurtzite and cubic mixed phases. With increasing molybdenum doping there is no change in the predominant wurtzite phase, but the peak becomes broad and unsymmetric.

The $a, c$ values are evaluated by the following formula [13]:

$$
\begin{array}{r}
\sin ^{2} \theta=\frac{\lambda}{3 a^{2}}\left(h^{2}+h k+k^{2}\right)+\frac{\lambda^{2}}{4 c^{2}}, \\
a=\frac{\lambda}{\sqrt{ } 3} \sin \theta .
\end{array}
$$

It is also observed that the stress develops in $\mathrm{ZnO}$ films due to Mo doping and is estimated from XRD spectra of the films.

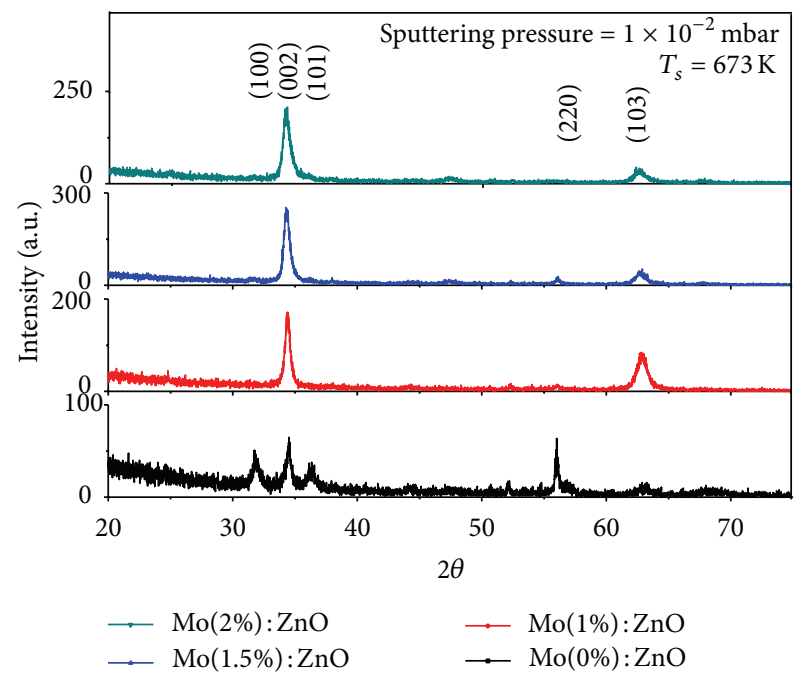

Figure 2: X-ray diffraction spectra of the Mo: $\mathrm{ZnO}$ thin films deposited at a fixed sputtering pressure of $1 \times 10^{-2}$ mbar and at a substrate temperature of $673 \mathrm{~K}$.

In case of highly $c$-axis oriented hexagonal crystals, the inplane stress $(\sigma)$ can be calculated based on the biaxial strain model [14]:

$$
\sigma=\left[2 C_{13}-\frac{C_{33}\left(C_{11}+C_{12}\right)}{C_{13}}\right] \frac{\left(c-c_{0}\right)}{c_{0}} .
$$

Here " $c$ " is the lattice parameter of the (002) reflection in the XRD spectra. $c_{0}$ is the corresponding bulk value (5.21 $\AA$ ), and $C_{i j}$ is elastic stiffness constant. $\left(C_{11}=2.1 \times 10^{11} \mathrm{~N} / \mathrm{m}^{2}\right.$, $C_{33}=2.1 \times 10^{11} \mathrm{~N} / \mathrm{m}^{2}, C_{12}=1.2 \times 10^{11} \mathrm{~N} / \mathrm{m}^{2}$, and $C_{13}=1.05$ $\times 10^{11} \mathrm{~N} / \mathrm{m}^{2}$.)

This results in the following relation for the stress:

$$
\sigma=-4.5 \times 10^{11} \frac{\left(c-c_{0}\right)}{c_{0}}\left(\mathrm{~N} / \mathrm{m}^{2}\right)
$$

The negative sign indicates that the Mo: $\mathrm{ZnO}$ films are in a state of compressive stress.

The total stress in the films commonly consists of two components. One is the intrinsic stress induced by the impurities and defects in the crystal, and the other is the extrinsic stress introduced by the lattice mismatch and the thermal expansion coefficient mismatch between the film and the substrate. The later component is negligible compared to the stress measured in the former $[15,16]$ when the films were deposited at lower substrate temperatures. 
In the present case, the intrinsic stress, that is, compressive stress, arises due to Mo. Depending on the size of the foreign impurity atom and the size of the host atom, the compressive stress may increase or decrease.

The compressive stress $(-\sigma)$ of the $\mathrm{Mo}(0 \%): \mathrm{ZnO}$ films deposited at $1 \times 10^{-2} \mathrm{mbar}$ and at $473 \mathrm{~K}$ is $4 \times 10^{10} \mathrm{~N} / \mathrm{m}^{2}$, and it decreases with increasing molybdenum concentration. The size of the $\mathrm{Mo}^{6+}$ ion is $(0.41 \AA)$, and it is low compared to that of $\mathrm{Zn}^{2+}$ ion $(0.60 \AA)$. When $\mathrm{Mo}$ is doped to $\mathrm{ZnO}$, some of the $\mathrm{Zn}$ atoms are substituted by Mo atoms. This substitution reduces the lattice parameter " $c$ " and further reduces the stress in the films. The similar type of behaviour was also observed in the case of $\mathrm{Al}$ doped $\mathrm{ZnO}$ films [17].

The compressive stress $(-\sigma)$ of the $\mathrm{Mo}(0 \%): \mathrm{ZnO}$ films deposited at $673 \mathrm{~K}$ is $-9.8 \times 10^{9} \mathrm{~N} / \mathrm{m}^{2}$. The $2 \theta$ of the (002) diffraction peak shifts towards lower value of $2 \theta$ with increasing molybdenum concentration in case of films deposited at $673 \mathrm{~K}$. This indicates the elongation of $c$-axis lattice constant which means that there is a residual stress in the plane of the films. The stress increases with increasing Mo concentration not only due to the elongation of $c$-axis lattice constant but also due to the thermal stress [1] generated by the films deposited at higher substrate temperatures. This conclusion is clearly supported by single optical transition observed in the optical transmittance spectra, and also a shift (MossBurstein) in the absorption edge is observed in the films doped with 1.5 and 2 at.\% molybdenum. So even the films contain appropriate at.\% of Mo which replaces the $\mathrm{Zn}$, the compressive stress is not reduced due to the thermal stress generated when the films are deposited at higher substrate temperatures. The variation of compressive stress $(-\sigma)$ of the Mo:ZnO films deposited at $1 \times 10^{-2}$ mbar and at different substrate temperatures of $473 \mathrm{~K}$ and $673 \mathrm{~K}$ is shown in Figure 3.

3.3. Surface Morphological Studies. The substrate temperature and $\mathrm{Ar}+\mathrm{O}_{2}$ partial pressures both effect the surface microstructure of undoped and molybdenum doped $\mathrm{ZnO}$ thin films. The surface microstructure of $\mathrm{ZnO}$ and $\mathrm{Mo}: \mathrm{ZnO}$ films was studied by FIB SEM images. Figures 4 and 5 show the surface microstructure of the films deposited at $1 \times$ $10^{-2} \mathrm{mbar}, 473 \mathrm{~K}$, and for 1 at.\% and 1.5 at.\% Mo. The 1 at.\% Mo content films show spherical particles of size $38 \mathrm{~nm}$ and are uniform, dense, and closely packed. With increasing at.\% of Mo, the grain size is increased to $45 \mathrm{~nm}$ due to segregation, and small grains are joined with each other and cause the elongation of grains rather than the forming of big grain. This is believed to be due to segregations caused by the increase in at.\% of Mo in film.

The grain size " $d$ " can also be derived from XRD using the Debye-Scherer formula:

$$
d=\frac{K \lambda}{\beta} \cos \theta
$$

where $K$ is the correction factor and is equal to 0.9 , and $\lambda$ is the X-ray wavelength $1.54 \AA$.

We have chosen (002) diffraction peak to calculate the grain size since it is the predominant peak in the XRD

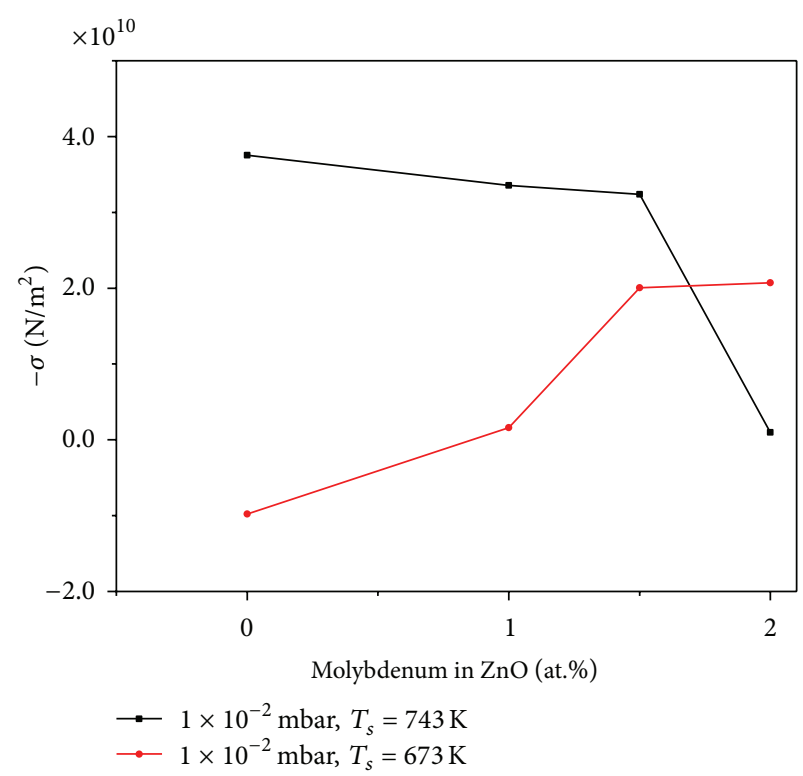

FIGURE 3: The variation of compressive stress in the Mo: $\mathrm{ZnO}$ films deposited at a sputtering pressure of $1 \times 10^{-2}$ mbar and at substrate temperatures of $473 \mathrm{~K}$ and $673 \mathrm{~K}$.

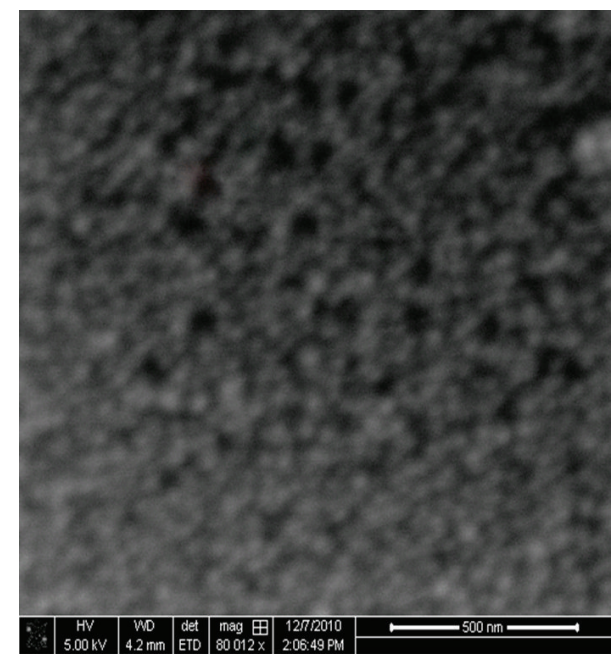

FIGURE 4: FIBSEM micrographs of $\mathrm{Mo}(1 \%): \mathrm{ZnO}$ films deposited at a sputtering pressure of $1 \times 10^{-2} \mathrm{mbar}$ and at a substrate temperature of $473 \mathrm{~K}$

spectra of the films deposited at all conditions. The grain size obtained from SEM is larger when compared with that determined from XRD. This difference is mainly caused by the different grain size criteria, underlying the different methods. Similar behavior was also observed in the case of $\mathrm{Al}$ doped $\mathrm{ZnO}$ films [17]. For uniformity, the grain sizes mentioned below were estimated by XRD. As the Mo content increases, the grain size increases for the films deposited at $1 \times 10^{-2} \mathrm{mbar}$ and at $473 \mathrm{~K}$. The reverse result in grain size variation was observed in case of films deposited at $673 \mathrm{~K}$ [18].

The literature reveals that the grain becomes small with the addition of impurities to the matrix material due to the induced defects and imperfection that behave as the source 


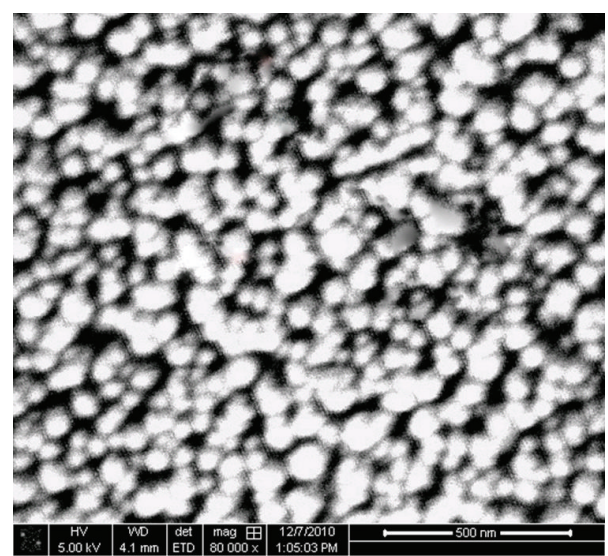

FIgURE 5: FIBSEM micrographs of $\mathrm{Mo}(1.5 \%): \mathrm{ZnO}$ films deposited at a sputtering pressure of $1 \times 10^{-2} \mathrm{mbar}$ and at a substrate temperature of $473 \mathrm{~K}$.

for forming grain boundaries $[9,19]$. These observations are also attributed to the correlation between the grain size and compressive stress of the films. As the stress decreases, the size increases. This indicates that the grain size $d$ is directly related to the stress in the film. The stress reduction plays two roles: (1) it diminishes the density of stress-induced defects in films, and improves the crystal perfection, and (2) it behaves as a mechanism, replacing the formation of islands [20] to relieve the strained system, and thus it facilitates the two-dimensional growth. Both of the two roles have beneficial effects on the grain growth. These two competitive processes, one for the conventional impurity mechanism and the another for the stress release mechanism, are probably the reasons that contribute to the observed change of grain sizes.

3.4. Optical Properties. The optical transmittance spectra of the films were analyzed to understand the type of optical transition and band structure and defects. The optical properties of $\mathrm{ZnO}$ and $\mathrm{Mo}: \mathrm{ZnO}$ films are influenced by oxygen and argon partial pressure, substrate temperatures, and Mo composition. The optical transmittance spectra of the Mo:ZnO films deposited at $1 \times 10^{-2} \mathrm{mbar}$ and at the two substrate temperatures of $473 \mathrm{~K}$ and $673 \mathrm{~K}$ are shown in Figures 6 and 7.

The optical transmittance of the films deposited at $1 \times$ $10^{-2}$ mbar and at $473 \mathrm{~K}$ decreases with increasing molybdenum concentration. The optical transmittance of the films decreases sharply near $400 \mathrm{~nm}$ due to the bandgap absorption. The absorption edge shifts towards lower wavelength with increasing molybdenum concentration. The energy gap of the films is found by the following formula [21]

$$
\alpha h v=B\left(h v-E_{g}\right)^{n},
$$

where $\alpha$ is the absorption coefficient, $v$ is the frequency of the incident light, $E_{g}$ is the energy gap, and " $n$ " is exponent, by plotting the graph between $(\alpha h \nu)^{2}$ versus $h \nu$. The plots of $(\alpha h \nu)^{2}$ versus $h v$ are shown in Figure 8. The plot shows better fit for $n=1 / 2$ indicating the direct transition of electron in $\mathrm{ZnO}[22-24]$.

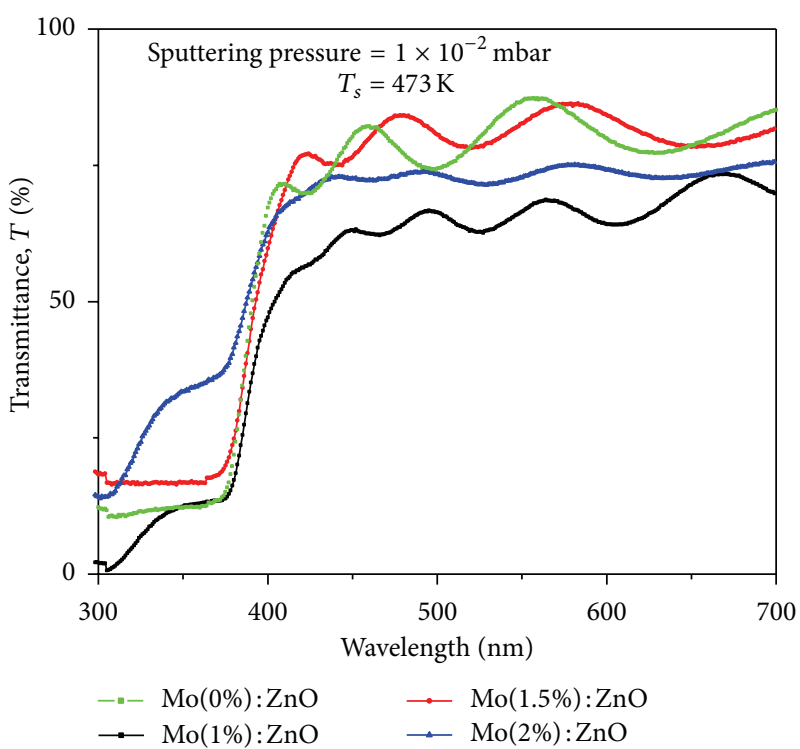

FIGURE 6: Optical transmittance spectra of $\mathrm{Mo:ZnO}$ thin films deposited at a sputtering pressure of $1 \times 10^{-2} \mathrm{mbar}$ and at a substrate temperature of $473 \mathrm{~K}$.

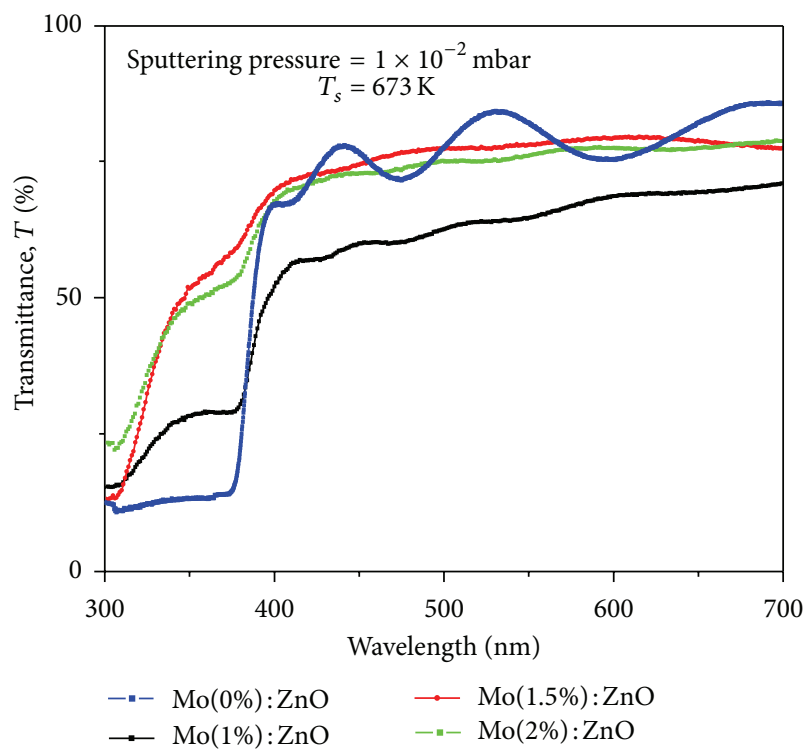

FIGURE 7: Optical transmittance spectra of $\mathrm{Mo:ZnO}$ thin films deposited at a sputtering pressure of $1 \times 10^{-2} \mathrm{mbar}$ and at a substrate temperature of $673 \mathrm{~K}$.

The energy gap of the films is $3.02 \mathrm{eV}$, and it increases with the increase of molybdenum concentration due to the Burstein-Moss shift [25]. Another transition at $350 \mathrm{~nm}$ was also observed, in case of films doped with 1 and 2 at.\% of Mo. This indicates that the films have two phases, that is, $\mathrm{MoO}_{3}$ and $\mathrm{Mo}: \mathrm{ZnO}$ which is due to the some percentage of molybdenum remains as $\mathrm{MoO}_{3}$. The optical transmittance of the films deposited at $1 \times 10^{-2} \mathrm{mbar}$ and at $673 \mathrm{~K}$ decreases with the increasing of molybdenum concentration, and the absorption edge shifts towards lower wavelength with increasing Mo concentration in $\mathrm{ZnO}$. 
TABle 2: Energy gap data of Mo: $\mathrm{ZnO}$ films.

\begin{tabular}{|c|c|c|c|c|}
\hline \multirow{4}{*}{ Atomic percent of $\mathrm{Mo}$ in $\mathrm{ZnO}$} & \multicolumn{4}{|c|}{ Sputtering pressure $=1 \times 10^{-2}$ mbar, } \\
\hline & \multicolumn{2}{|c|}{$T_{s}=473 \mathrm{~K}$} & \multicolumn{2}{|c|}{$T_{s}=673 \mathrm{~K}$} \\
\hline & 1st transition & 2nd transition & 1st transition & 2nd transition \\
\hline & \multicolumn{2}{|c|}{ Energy gap $\left(E_{g}\right)(\mathrm{eV})$} & \multicolumn{2}{|c|}{ Energy gap $\left(E_{g}\right)(\mathrm{eV})$} \\
\hline $\mathrm{Mo}(0 \%): \mathrm{ZnO}$ & 2.48 & 3.02 & - & 3.15 \\
\hline $\mathrm{Mo}(1 \%): \mathrm{ZnO}$ & - & 3.05 & 3.06 & 3.26 \\
\hline $\mathrm{Mo}(1.5 \%): \mathrm{ZnO}$ & 2.66 & 3.17 & - & 3.43 \\
\hline $\mathrm{Mo}(2 \%): \mathrm{ZnO}$ & - & 3.34 & - & 3.56 \\
\hline
\end{tabular}

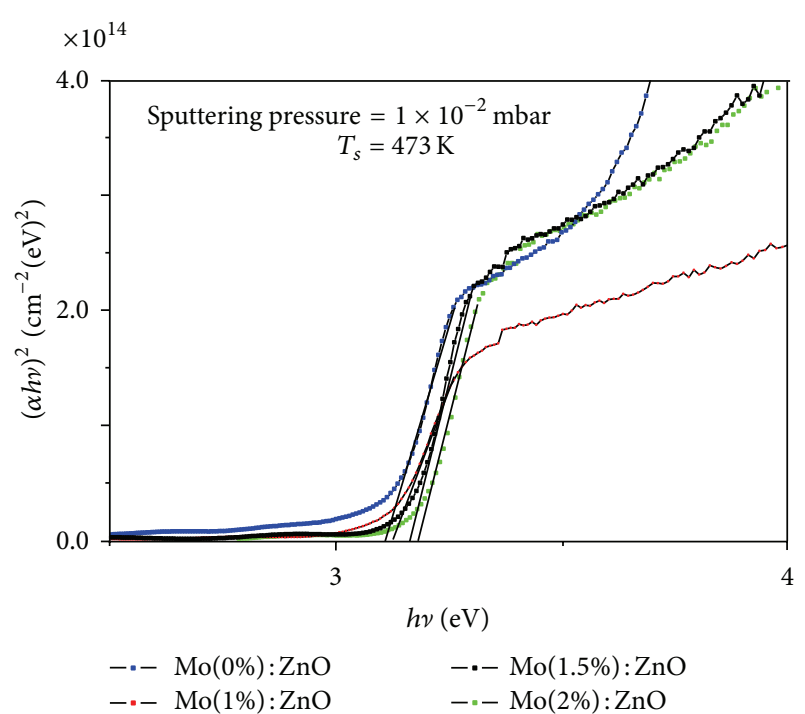

FIgURE 8: Plots of $(\alpha h \nu)^{2}$ versus $h v$ of Mo: $\mathrm{ZnO}$ thin films deposited at a fixed sputtering pressure of $1 \times 10^{-2} \mathrm{mbar}$ and at a substrate temperature $473 \mathrm{~K}$.

The multiple optical transitions (two absorption edges) observed above were also present in the case of Mo content of 1 at. $\%$ in $\mathrm{ZnO}$ due to the same reason as above. But at the same substrate temperature of $673 \mathrm{~K}$, the reactivity between $\mathrm{Mo}$ and $\mathrm{ZnO}$ in the vapour phase is improved and overcomes the multiple transitions in case of films doped with 1.5 and 2 at.\% Mo to $\mathrm{ZnO}$. The results were in good agreement with reported values $[2,26]$.

The variation of energy gap of $\mathrm{ZnO}$ films with $\mathrm{Mo}$ concentration is given in Table 2 .

\section{Conclusions}

Undoped and molybdenum doped $\mathrm{ZnO}$ thin films were deposited by r.f. magnetron sputtering, and their compositional, structural, surface morphological, and optical properties were studied. The composition study reveals that, at a fixed partial pressure, the substrate temperature and molybdenum concentration influence the composition, structure, surface morphology, and optical properties of the $\mathrm{ZnO}$ films.

The $\mathrm{ZnO}$ films deposited at $1 \times 10^{-2} \mathrm{mbar}$ and at $473 \mathrm{~K}$ have oxygen deficiency. The $\mathrm{Zn} / \mathrm{O}$ ratio increased with increasing Mo at.\%. The structure of $\mathrm{ZnO}$ films is hexagonal wurtzite with predominant (002) crystallographic orientation. There is no change in the crystal structure of the films with Mo doping. The compressive stress decreased with increasing Mo at.\% in $\mathrm{ZnO}$. The films deposited at $673 \mathrm{~K}$ have lower stress when compared to the films deposited at $473 \mathrm{~K}$. The grain size of the films deposited at $473 \mathrm{~K}$ increased with increasing $\mathrm{Mo}$ at.\% in $\mathrm{ZnO}$ and decreased when deposited at $673 \mathrm{~K}$. The surface morphology indicates that the films contain lower particle size when deposited at lower substrate temperatures and higher particle size when deposited at higher substrate temperatures. The optical transmittance spectra reveal that the Mo effectively dopes to $\mathrm{ZnO}$ when the films are deposited at higher substrate temperatures than at lower temperatures. In conclusion, Mo can be effectively doped to $\mathrm{ZnO}$ at optimized deposition conditions.

\section{Acknowledgments}

The authors are thankful to A. K. Ray Chaudhari, Director of SNBNCBS, for allowing them to do FIBSEM measurements and the technicians Piyali Bose, Pallab, and Shaktinath Das for their valuable help during the course of this work.

\section{References}

[1] J. Mass, P. Bhattacharya, and R. S. Katiyar, "Effect of high substrate temperature on $\mathrm{Al}$-doped $\mathrm{ZnO}$ thin films grown by pulsed laser deposition," Materials Science and Engineering B, vol. 103, no. 1, pp. 9-15, 2003.

[2] S. S. Lin, J. L. Huang, and P. Šajgalik, "The properties of heavily Al-doped $\mathrm{ZnO}$ films before and after annealing in the different atmosphere," Surface \& Coating Technologies, vol. 185, no. 2-3, pp. 254-263, 2004.

[3] E. L. Papadopoulou, M. Varda, K. Kouroupis-Agalou et al., "Undoped and Al-doped $\mathrm{ZnO}$ films with tuned properties grown by pulsed laser deposition," Thin Solid Films, vol. 516, no. 22, pp. 8141-8145, 2008.

[4] J.-H. Lee and B.-O. Park, "Transparent conducting ZnO:Al, In and Sn thin films deposited by the sol-gel method," Thin Solid Films, vol. 426, no. 1-2, pp. 94-99, 2003.

[5] H. K. Kim, S. H. Huh, J. W. Park, J. W. Jeong, and G. H. Lee, "The cluster size dependence of thermal stabilities of both molybdenum and tungsten nanoclusters," Chemical Physics Letters, vol. 354, no. 1-2, pp. 165-172, 2002.

[6] J. N. Duenow, T. A. Gesset, D. M. Wood, T. M. Barnes, and M. Young, "Transparent conducting zinc oxide thin films doped 
with aluminum and molybdenum," Journal of Vacuum Science \& Technology, vol. 25, no. 4, p. 955, 2007.

[7] J. Shi, H. Ma, G. Ma, H. Ma, and J. Shen, "Structure and ultrafast carrier dynamics in n-type transparent $\mathrm{Mo}: \mathrm{ZnO}$ nanocrystalline thin films," Applied Physics, vol. 92, no. 2, pp. 357-360, 2008.

[8] Y. C. Lin, B. L. Wang, W. T. Yen, C. T. Ha, and C. Peng, "Effect of process conditions on the optoelectronic characteristics of ZnO:Mo thin films prepared by pulsed direct current magnetron sputtering," Thin Solid Films, vol. 518, no. 17, pp. 49284934, 2010.

[9] D. J. Cohen, K. C. Ruthe, and S. A. Barnett, "Transparent conducting Znl-xMgxO:(Al,In) thin films," Journal of Applied Physics, vol. 96, no. 1, pp. 459-467, 2004.

[10] K. S. Rao, B. Rajini Kanth, K. Pandurangarao, and P. K. Mukhopadhyay, "Physical investigations on pulsed laser deposited nanocrystalline $\mathrm{ZnO}$ thin films," Applied Physics A, vol. 108, no. 1, pp. 247-254, 2012.

[11] A. Manthiram and J. Gopalakrishnan, "New $\mathrm{A}^{2+} \mathrm{Mo}^{4+} \mathrm{O}_{3}$ oxides with defect spinel structure," Materials Research Bulletin, vol. 15, no. 2, pp. 207-211, 1980.

[12] R. Swapna and M. C. Santhosh Kumar, "The role of substrate temperature on the properties of nanocrystalline Mo doped $\mathrm{ZnO}$ thin films by spray pyrolysis," Ceramics International, vol. 38, no. 5, pp. 3875-3883, 2012.

[13] B. D. Cullity, Elements of X-Ray Diffraction, Addison-Wesley, Philippines, 2nd edition, 1977.

[14] S. Maniv, W. D. Westwood, and E. Colombini, "Pressure and angle of incidence effects in reactive planar magnetron sputtered ZnO layers," Journal of Vacuum Science \& Technology, vol. 20, no. 2, pp. 162-170, 1982.

[15] R. Cebulla, R. Wendt, and K. Ellmer, "Al-doped zinc oxide films deposited by simultaneous $\mathrm{rf}$ and dc excitation of a magnetron plasma: relationships between plasma parameters and structural and electrical film properties," Journal of Applied Physics, vol. 83, no. 2, pp. 1087-1095, 1998.

[16] T. Hanabusa, H. Hosoda, K. Kusaka, and K. Tominaga, "Abnormal residual stress state in $\mathrm{ZnO}$ films synthesized by planar magnetron sputtering system with two facing targets," Thin Solid Films, vol. 343-344, no. 1-2, pp. 164-167, 1999.

[17] J. G. Lu, Z. Z. Ye, Y. J. Zeng et al., "Structural, optical, and electrical properties of $(\mathrm{Zn}, \mathrm{Al}) \mathrm{O}$ films over a wide range of compositions," Journal of Applied Physics, vol. 100, no. 7, Article ID 073714, 11 pages, 2006.

[18] Y. C. Lin, B. L. Wang, W. T. Yen, and C. H. Shen, "Surface textured molybdenum doped zinc oxide thin films prepared for thin film solar cells using pulsed direct current magnetron sputtering," Thin Solid Films, vol. 519, no. 16, pp. 5571-5576, 2011.

[19] P. Nunes, E. Fortunato, P. Tonello, F. Braz Fernandes, P. Vilarinho, and R. Martins, "Effect of different dopant elements on the properties of $\mathrm{ZnO}$ thin films," Vacuum, vol. 64, no. 3-4, pp. 281-285, 2002.

[20] D. J. Eaglesham, F. C. Unterwald, and D. C. Jacobson, "Growth morphology and the equilibrium shape: the role of surfactants in Ge/Si island formation," Physical Review Letters, vol. 70, no. 7, pp. 966-969, 1993.

[21] M. Green and A. Travlas, "Sodium-tungsten bronze thin films: I. Optical properties of dilute bronzes," Philosophical Magazine, vol. 51, no. 5, pp. 501-520, 1985.

[22] M. Suchea, S. Christoulakis, M. Katharakis, N. Vidakis, and E. Koudoumas, "Influence of thickness and growth temperature on the optical and electrical properties of $\mathrm{ZnO}$ thin films," Thin Solid Films, vol. 517, no. 15, pp. 4303-4306, 2009.

[23] F. K. Shan, G. X. Liu, W. J. Lee, and B. C. Shin, "Stokes shift, blue shift and red shift of $\mathrm{ZnO}$-based thin films deposited by pulsedlaser deposition," Journal of Crystal Growth, vol. 291, no. 2, pp. 328-333, 2006.

[24] S. Logothetidis, A. Laskarakis, S. Kassavetis, S. Lousinian, C. Gravalidis, and G. Kiriakidis, "Optical and structural properties of $\mathrm{ZnO}$ for transparent electronics," Thin Solid Films, vol. 516, no. 7, pp. 1345-1349, 2008.

[25] E. Burstein, "Anomalous optical absorption limit in InSb," Physical Review, vol. 93, no. 3, pp. 632-633, 1954.

[26] A. Boukhachem, B. Ouni, M. Karyaoui, A. Madani, R. Chtourou, and M. Amlouk, "Structural, opto-thermal and electrical properties of $\mathrm{ZnO}$ :Mo sprayed thin films," Materials Science in Semiconductor Processing, vol. 15, no. 3, pp. 282-292, 2012. 

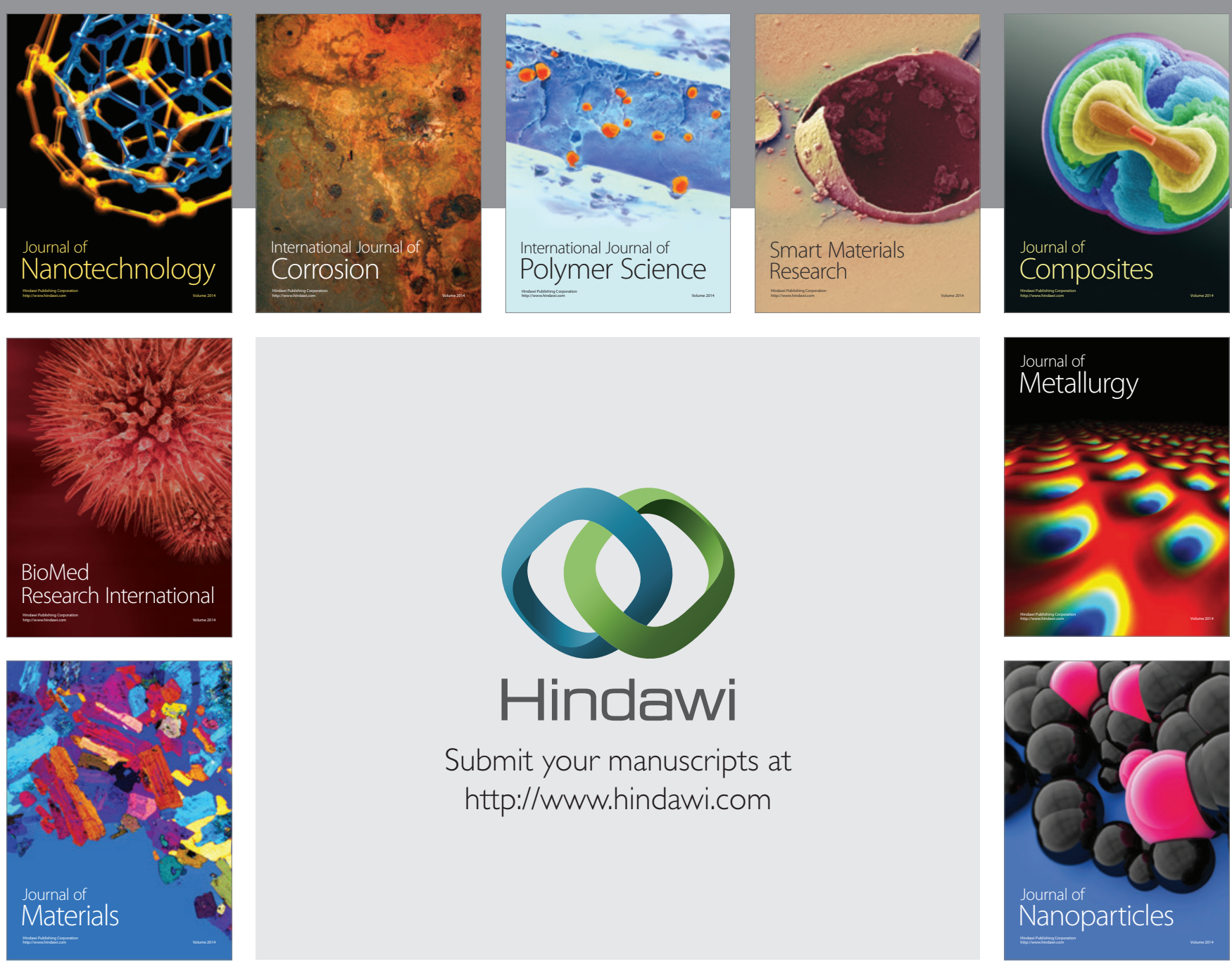

Submit your manuscripts at http://www.hindawi.com
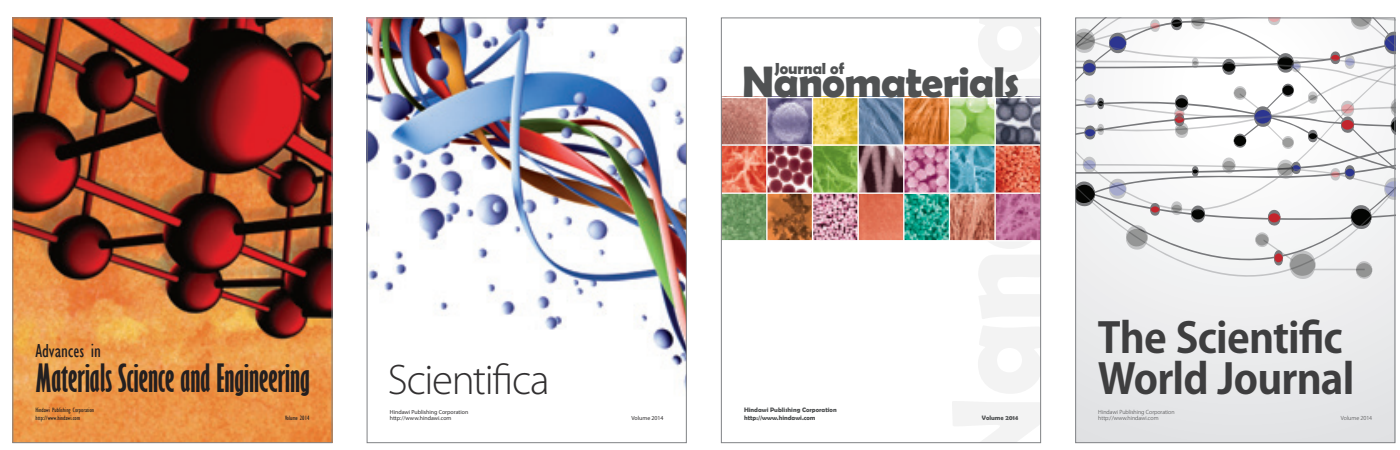

\section{The Scientific World Journal}
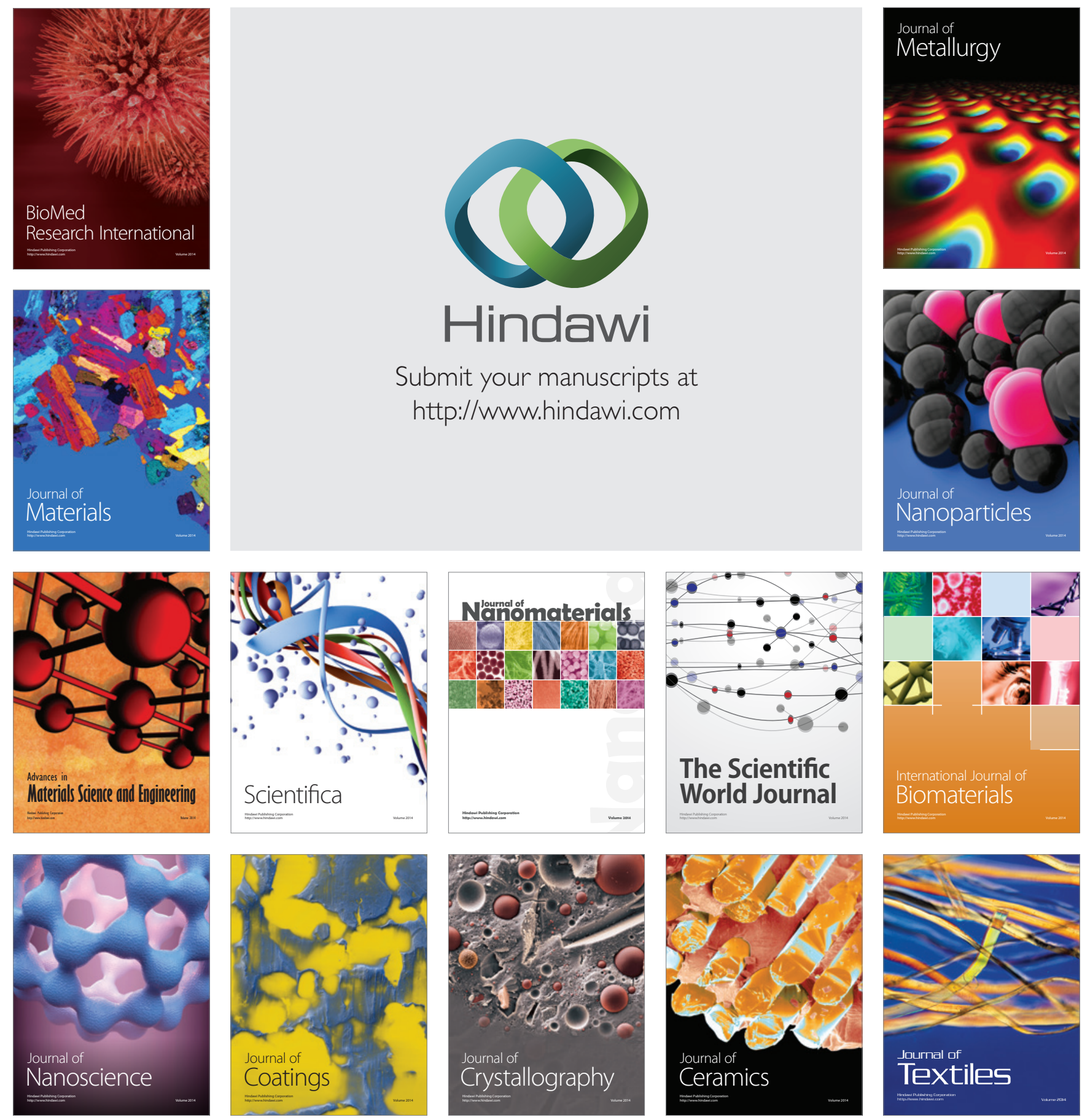